\title{
Modeling and $\boldsymbol{H}_{\infty}$ Robust Control of a Smart Structure with Rate-dependent Hysteresis Nonlinearity
}

\author{
Ping Liu ${ }^{1} \quad$ Zhen-Yan Wang ${ }^{1}$ Zhen Zhang ${ }^{1}$ Jian-Qin Mao ${ }^{1}$ Ke-Min Zhou ${ }^{2,3}$ \\ ${ }^{1}$ School of Automation Science and Electrical Engineering, Beijing University of Aeronautics \& Astronautics, Beijing 100191, China \\ ${ }^{2}$ School of Electrical Engineering, Southwest Jiaotong University, Chengdu, Sichuan 610031, China \\ ${ }^{3}$ School of Electrical Engineering and Computer Science, Louisiana State University, Baton Rouge, LA 70803, USA
}

\begin{abstract}
The performance of smart structures in trajectory tracking under sub-micron level is hindered by the rate-dependent hysteresis nonlinearity. In this paper, a Hammerstein-like model based on the support vector machines (SVM) is proposed to capture the rate-dependent hysteresis nonlinearity. We show that it is possible to construct a unique dynamic model in a given frequency range for a rate-dependent hysteresis system using the sinusoidal scanning signals as the training set of signals for the linear dynamic subsystem of the Hammerstein-like model. Subsequently, a two-degree-of-freedom (2DOF) $H_{\infty}$ robust control scheme for the ratedependent hysteresis nonlinearity is implemented on a smart structure with a piezoelectric actuator (PEA) for real-time precision trajectory tracking. Simulations and experiments on the structure verify both the effectiveness and the practicality of the proposed modeling and control methods.
\end{abstract}

Keywords: Rate-dependent hysteresis, Hammerstein-like model, support vector machines (SVM), $H_{\infty}$ robust control, piezoelectric actuator.

\section{Introduction}

The piezoelectric actuators (PEA) have received tremendous interests in the past two decades and have been widely used as micro-positioners, vibration controllers, sonar projectors and insulators, etc. The PEA applied in such devices displays prominent capabilities of high stiffness, fast frequency response and high resolution.

However, wider applications of the piezoelectric actuators are restricted due to an inherent property of the piezoelectric materials, the hysteresis nonlinearity, which can cause undesirable inaccuracy, oscillation or even instability of the systems. The hysteresis is embodied as a nonlinear relationship between the input voltage and the output displacement, and is dependent not only on the amplitude but also on the frequencies of the input signals. Hence the hysteresis system is rate-dependent ${ }^{[1]}$. As shown in Fig. 1, the shape of the hysteresis loops changes significantly with the frequencies of the input signals. So it is a major challenge to capture the complicated rate-dependent hysteretic behavior precisely. Then the modeling and control of the ratedependent hysteresis have attracted much attention for its sophisticated nature and extensive applications.

The existing techniques for the rate-dependent hysteresis modeling can be classified into three categories. The first category is based on phenomenological models. Recent propositions include the modified Preisach model ${ }^{[2]}$, the modified Prandtl-Ishlinskii model ${ }^{[3]}$, and the generalized Prandtl-Ishlinskii model ${ }^{[4]}$. The second category contains the physical models. For instance, Oh and Bernstein ${ }^{[5,6]}$ developed the semilinear Duhem model to describe the rate-

\footnotetext{
Manuscript received July 6, 2012; revised July 1, 2013

This work was supported by National Natural Science Foundation of China (Nos.91016006 and 91116002), Fundamental Research Funds for the Central Universities (Nos. 30420111109, 30420120305 and SWJTU11ZT06), and in part by a PFund from Louisiana Board of Regents.
}

dependent hysteresis. The third category is the intelligent methods, such as artificial neural network $(\mathrm{ANN})^{[7,8]}$, fuzzy tree $(\mathrm{FT})^{[9]}$, all providing efficient ways to model the ratedependent hysteresis. Control of smart structures with consideration of hysteresis nonlinearity has also received much attention. A very effective approach in dealing with the hysteresis in control system is to find an accurate model of the hysteresis and then to use inverse compensation to cancel out the hysteresis ${ }^{[10,11]}$. In addition, some feedback control schemes including adaptive control ${ }^{[12]}$, robust control ${ }^{[13]}$, and optimal control ${ }^{[14]}$ were also adopted in the controller synthesis.

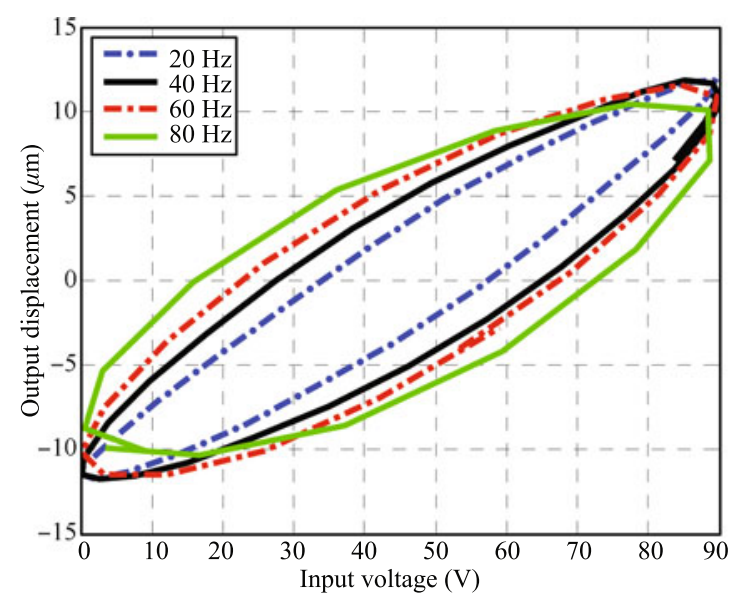

Fig. 1 Rate-dependent hysteresis loops of PEA

In this paper, we study the modeling and trajectory tracking control of a PEA with rate-dependent hysteresis nonlinearity under variable frequencies of input signals. First of all, a Hammerstein-like configuration ${ }^{[15,16]}$, which includes a nonlinear dynamic block followed by a linear 
dynamic block, is employed as a universal model to capture the rate-dependent hysteresis. The paper describes a method for identifying the nonlinear dynamic block of the Hammerstein-like system based on support vector machines (SVM), and further gives estimates of the parameters governing the linear dynamic block represented as an autoregressive exogenous (ARX) model. Then, an $H_{\infty}$ robust control scheme based on the proposed hysteresis model is applied to the PEA for real-time trajectory tracking control. Experiments are carried out to validate the proposed model and the control scheme.

\section{Rate-dependent hysteresis model}

\subsection{Hammerstein-like model}

It is well known that finding an exact model for a complex nonlinear system is usually very difficult and sometimes may not even be desirable for control purpose due to the complexity of the model. However, the Hammerstein type of models, may be good approximations for some classes of complex nonlinear systems. A Hammerstein model is composed of a memoryless static nonlinearity followed by a linear dynamical system.

Extensive research work has been done on the parametric model identification of the Hammerstein models. Existing identification methods for the Hammerstein systems include the over-parameterization method ${ }^{[17]}$, the subspace method $^{[18]}$, the separable least squares approach ${ }^{[19]}$, the blind method ${ }^{[20]}$, the iterative method ${ }^{[21]}$ and other identification methods.

However, the conventional Hammerstein model is restricted to the situation where the hysteresis loop changes with the frequency of the input-signal while the nonlinear part remains fairly static. To overcome this drawback, the Hammerstein-like model, which has the same structure as the Hammerstein model but involves dynamic nonlinearity, is mentioned in [22]. The rate-dependent hysteresis model shown in Fig. 2 is a Hammerstein-like model, which is the focus of this paper.

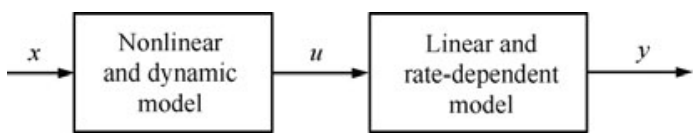

Fig. 2 Structure of a rate-dependent Hammerstein-like hysteresis model

We propose a nonlinear identification method based on the support vector machines (SVM) and model the linear part as an ARX model with a $p$-th order numerator and a $q$-th order denominator transfer function. Specifically, the ARX model is assumed to have the following form:

$$
y_{t}=\sum_{i=1}^{q} a_{i} y_{t-i}+\sum_{j=0}^{p} b_{i} u_{t-j}+e_{t}, \quad \forall t \in \mathbf{z}
$$

where the equation error $e_{t}$ is assumed to be white and zero mean and the parameters of the ARX model are denoted by $\theta=\left(a_{1}, \cdots, a_{q}, b_{0}, \cdots, b_{p}\right)^{\mathrm{T}} \in \mathbf{R}^{p+q+1}$.

\subsection{SVM for function approximation}

SVM based on the Vapnik-Chervonenkis (VC) dimension theory and the structural risk minimization principal are developed by Vapnik $^{[23]}$ to solve classification and regression problems. The basic concept of the SVM for regression (SVR) is to map nonlinearly the original data into a higher dimensional feature space. Hence, given a set of data $\left\{\left(x_{k}, y_{k}\right)\right\}_{k=1}^{N}$ with input data $x_{k} \in \mathbf{R}^{n}$ and output data $y_{k} \in \mathbf{R}$, the SVR function is

$$
f(x)=\langle\omega, \varphi(x)\rangle+b
$$

where $\langle\cdot, \cdot\rangle$ denotes the dot product, $\varphi(\cdot): \mathbf{R}^{n} \rightarrow \mathbf{R}^{n_{h}}$ is the mapping to the high dimensional and potentially infinite dimensional feature space, $\omega \in \mathbf{R}^{n_{h}}$ is the weight vector in primal weight space, $b \in \mathbf{R}$ is the bias term. The $\omega$ and $b$ are estimated by minimizing the regularized risk function given by

$$
R=\frac{1}{2}\|\omega\|^{2}+C \sum_{k=1}^{N}\left|y_{k}-f\left(x_{i}\right)\right|_{\varepsilon}
$$

where $|y-f(x)|_{\varepsilon}=\max \{0,|y-f(x)|-\varepsilon\}$ is called the $\varepsilon$ insensitive loss function, $C$ and $\varepsilon$ are prescribed parameters, $\frac{\|\omega\|^{2}}{2}$ measures the flatness of the function. Therefore, $C$ is considered to specify the trade-off between the empirical risk and the model flatness.

Two positive slack variables $\zeta$ and $\zeta^{*}$, which represent the distance from actual values to the corresponding boundary values of $\varepsilon$-tube, are introduced. Equation (3) is transformed into the following constrained form

$$
\begin{gathered}
\min _{J}\left(\omega, \zeta, \zeta^{*}\right)=\frac{1}{2}\|\omega\|^{2}+C \sum_{k=1}^{N}\left(\zeta_{k}+\zeta_{k}^{*}\right) \\
\left\langle\omega, \varphi\left(x_{k}\right)\right\rangle+b_{k}-y_{k} \leqslant \varepsilon+\zeta_{k} \\
\text { s.t. } \quad y_{k}-\left\langle\omega, \varphi\left(x_{k}\right)\right\rangle-b_{k} \leqslant \varepsilon+\zeta_{k}^{*} \\
\quad \zeta_{k}, \zeta_{k}^{*} \geqslant 0, k=1,2, \cdots, N .
\end{gathered}
$$

This constrained optimization problem is solved using the following primal Lagrangian form:

$$
\begin{aligned}
& L\left(\omega, b, \zeta, \zeta^{*}, \alpha_{k}, \alpha_{k}^{*}, \eta_{k}, \eta_{k}^{*}\right)= \\
& \quad \frac{1}{2}\|\omega\|^{2}+C \sum_{k=1}^{N}\left(\zeta_{k}+\zeta_{k}^{*}\right)-\sum_{k=1}^{N}\left(\eta_{k} \zeta_{k}+\eta_{k}^{*} \zeta_{k}^{*}\right)- \\
& \sum_{k=1}^{N} \alpha_{k}\left[\varepsilon+\zeta_{k}-\left\langle\omega, \varphi\left(x_{k}\right)\right\rangle-b_{k}+y_{k}\right]- \\
& \sum_{k=1}^{N} \alpha_{k}^{*}\left[\varepsilon+\zeta_{k}^{*}+\left\langle\omega, \varphi\left(x_{k}\right)\right\rangle+b_{k}-y_{k}\right] .
\end{aligned}
$$

Equation (5) is minimized with respect to primal variables $\omega, b, \zeta$ and $\zeta^{*}$, and maximized with respect to nonnegative Lagrangian multipliers $\alpha, \alpha^{*}, \eta$ and $\eta^{*}$. Therefore, (6) is obtained as

$$
\left\{\begin{array}{l}
\frac{\partial L}{\partial \omega}=0 \rightarrow \omega=\sum_{k=1}^{N}\left(\alpha_{k}^{*}-\alpha_{k}\right) \varphi\left(x_{k}\right) \\
\frac{\partial L}{\partial b}=0 \rightarrow \sum_{k=1}^{N}\left(\alpha_{k}^{*}-\alpha_{k}\right)=0 \\
\frac{\partial L}{\partial \zeta_{k}}=0 \rightarrow C-\alpha_{k}-\eta_{k}=0 \\
\frac{\partial L}{\partial \zeta_{k}^{*}}=0 \rightarrow C-\alpha_{k}^{*}-\eta_{k}^{*}=0
\end{array}\right.
$$


Finally, Karush-Kuhn-Tucker (KKT) conditions are applied to the regression, and (4) thus yields the dual Lagrangian by substituting (6) into (5). Then, the dual Lagrangian (7) is obtained by using kernel function $K\left(x_{k}, x_{l}\right)=\phi\left(x_{k}\right) \phi\left(x_{l}\right)$.

$$
\begin{aligned}
& \max \left\{W\left(\alpha_{k}, \alpha_{k}^{*}\right)\right\}=\sum_{k=1}^{N}\left(\alpha_{k}-\alpha_{k}^{*}\right) y_{k}-\varepsilon \sum_{k=1}^{N}\left(\alpha_{k}+\alpha_{k}^{*}\right)- \\
& \quad \frac{1}{2} \sum_{k=1}^{N} \sum_{l=1}^{N}\left(\alpha_{k}-\alpha_{k}^{*}\right)\left(\alpha_{l}-\alpha_{l}^{*}\right) K\left(x_{k}, x_{l}\right) \\
& \text { s.t. } \sum_{k=1}^{N}\left(\alpha_{k}-\alpha_{k}^{*}\right)=0, \quad 0 \leqslant \alpha_{k}, \alpha_{k}^{*} \leqslant C, \quad k=1, \cdots, N .
\end{aligned}
$$

The Lagrange multipliers in (7) satisfy the equality $\alpha \times \alpha^{*}=0$. The Lagrange multipliers $\alpha$ and $\alpha^{*}$ are calculated and a desired optimal weight vector of the regression hyperplane is

$$
\omega^{*}=\sum_{k=1}^{N}\left(\alpha_{k}-\alpha_{k}^{*}\right) \varphi\left(x_{k}\right) .
$$

Hence, the regression estimate function is

$$
f(x)=\sum_{k=1}^{N}\left(\alpha_{k}-\alpha_{k}^{*}\right) K\left(x, x_{k}\right)+b
$$

where $K\left(x, x_{k}\right)$ is called the kernel function. In this work, the radial basis function $(\mathrm{RBF})$ is used.

$$
K\left(x, x_{k}\right)=\mathrm{e}^{\left(\frac{-\left\|x-x_{k}\right\|^{2}}{\sigma^{2}}\right)}
$$

where $\sigma$ denotes the bandwidth of the kernel and $\|\cdot\|$ represents the Euclidean distance. Then the parameters that users have to specify are the error goal $\varepsilon$, the constant $C$ and the width of the RBF $\sigma$.

\subsection{Parameters identification}

Because the particle swarm optimization (PSO) algorithm has a special ability to simplify the optimization implementation and to quickly converge to a good solution, automatic parameters selection for SVM based on PSO is proposed in this paper.

PSO is a computational intelligence technique, which was motivated by the organisms' behavior such as shoaling of fish and flocking of birds. PSO can solve a variety of difficult optimization problems. The major advantage of PSO is that it employs the physical movements of the individuals in the swarm and has a flexible and well-balanced mechanism to enhance and adapt to the global and local exploration abilities. Another advantage is its simplicity in coding and consistency in performance ${ }^{[24]}$.

The selection of three parameters $(\varepsilon, C$ and $\sigma$ ) of an SVR model is important to the generalization of the Hammerstein-like model. The PSO algorithm is used to optimize the parameters for improving the precision. In the PSO algorithm, the parameters $\varepsilon, C$ and $\sigma$ become the particles. Each particle can make use of the best position encountered by itself and that of its neighbors to position itself toward an optimal solution. The performance of each particle is evaluated using a predefined fitness function, which encapsulates the characteristics of the optimization problem. In this study, the smaller the value of fitness function is, the better the particle is.

In each iteration, every particle calculates its velocity and position according to

$$
\left\{\begin{aligned}
v_{i}^{k+1} & =\omega v_{i}^{k}+c_{1} r_{1}\left(p_{i}^{k}-x_{i}^{k}\right)+c_{2} r_{2}\left(p_{g}^{k}-x_{i}^{k}\right) \\
x_{i}^{k+1} & =x_{i}^{k}+v_{i}^{k+1}
\end{aligned}\right.
$$

where $k$ is the current step number, $\omega$ is the inertia weight, $c_{1}$ and $c_{2}$ are the acceleration constants, $r_{1}$ and $r_{2}$ are the two random numbers in the range $[0,1], x_{i}^{k}$ is the current position of the particle, $p_{i}^{k}$ is the best one of the solutions this particle has reached, $p_{g}^{k}$ is the best one of the solutions all the particles have reached.

The process of obtaining the optimal parameters by the PSO from the SVM model is described in the flowchart shown in Fig. 3.

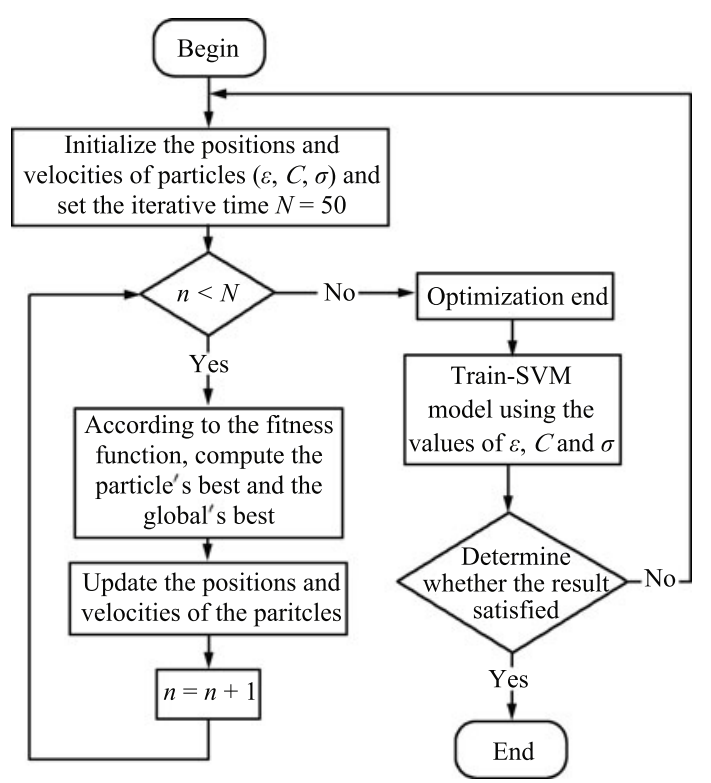

Fig. 3 The flowchart of optimizing the SVM parameters with PSO

\subsection{Modeling performance}

The modeling of rate-dependent Hammerstein-like hysteresis can be divided into two parts: The first part is to identify the nonlinear subsystem through the SVM method based on PSO. And the second part is to identify the linear subsystem through ARX approach, which represents the rate-dependent information in the whole model.

It is noted that our rate-dependent Hammerstein-like hysteresis model is not only valid for single-signal hysteresis loop but also for compound-signal hysteresis loop. The reason we consider the compound frequency input signal is that this kind of signal can excite more frequency information of PEA. And the compound frequency input signal can be obtained through the single-signals averaging technique.

The general approximation property of the SVM makes it possible to be used as a nonlinear dynamic system iden- 
tifier. In order to transform the multi-valued mapping of the hysteresis nonlinearity into a one-to-one mapping, a dynamic discrete framework is proposed as ${ }^{[25]}$

$$
\begin{aligned}
\hat{y}(k+1)= & f(x(k), x(k-1), \cdots, x(k-m) ; \\
& y(k), y(k-1), \cdots, y(k-n))
\end{aligned}
$$

where $x(k)$ and $y(k)$ are the input and true output of the system at time instant $k$, respectively, $\hat{y}(k+1)$ is the estimated output of the model at time instant $k+1, m$ and $n$ are the dynamic orders. The fundamental idea of the modeling method is to predict the output of the next step by utilizing the current and previous inputs and outputs of the plant.

The linear and rate-dependent subsystem can be represented as

$$
L=\frac{B(\mathrm{z})}{A(z)}
$$

where $A(z)$ and $B(z)$ are polynomials in the shift operator $z^{-1}$ with $z^{-1} y(t)=y(t-1)$, and

$$
\left\{\begin{array}{l}
A(z)=1+a_{1} z^{-1}+a_{2} z^{-2}+\ldots+a_{q} z^{-q} \\
B(z)=b_{0}+b_{1} z^{-1}+b_{2} z^{-2}+\ldots+b_{p} z^{-p}
\end{array}\right.
$$

where $q$ and $p$ are the orders of the polynomials of $A(z)$ and $B(z)$. The optimal values of $p$ and $q$ are searched based on Akaike's information criterion (AIC) which is a practical criterion to determine the orders of linear models ${ }^{[26]}$.

With the proposed model parameters identification methods, we get the parameters of the rate-dependent Hammerstein-like hysteresis model as

$$
\begin{gathered}
\varepsilon=0.001, \quad C=623458.24, \quad \sigma=785.64 \\
m=4, n=4, p=2, \quad q=2 .
\end{gathered}
$$

We first use a $1 \mathrm{~Hz}$ single-signal data to construct the nonlinear subsystem of the Hammerstein-like model through the SVM, then apply the sinusoidal scanning signals data from $1 \mathrm{~Hz}$ to $100 \mathrm{~Hz}$ to obtain the ARX model. Finally, we test the Hammerstein-like model using the singlesignal data from $1 \mathrm{~Hz}$ to $100 \mathrm{~Hz}$ and some sets of compoundsignal data, respectively.

Our experimental data are obtained at a sampling frequency of $10 \mathrm{kHz}$. At every frequency, we take 500 pairs from all experimental data and the model simulation. The modeling and tracking control results can all be evaluated through the root mean square error (RMSE) and the relative error (RE), which are defined, respectively, as

$$
\begin{aligned}
\mathrm{RMSE} & =\sqrt{\frac{\sum_{i=1}^{N}\left|y_{i}-y_{i}^{\prime}\right|^{2}}{N}} \\
\mathrm{RE} & =\sqrt{\frac{\sum_{i=1}^{N}\left|y_{i}-y_{i}^{\prime}\right|^{2}}{\sum_{i=1}^{N}\left|y_{i}\right|^{2}}}
\end{aligned}
$$

where $N$ is the number of data, $y_{i}$ is the experimental output, and $y_{i}^{\prime}$ is the output calculated from the model.
Fig. 4 and Table 1 show the comparison between the ratedependent hysteresis loop measured by experiments and those simulated based on the Hammerstein-like modeling method at several single-signal and compound-signal inputs.

From the results in Fig. 4 and Table 1, we can see the outstanding applicability of the constructed ratedependent Hammerstein-like model for both single-signal and compound-signal hysteresis loops.
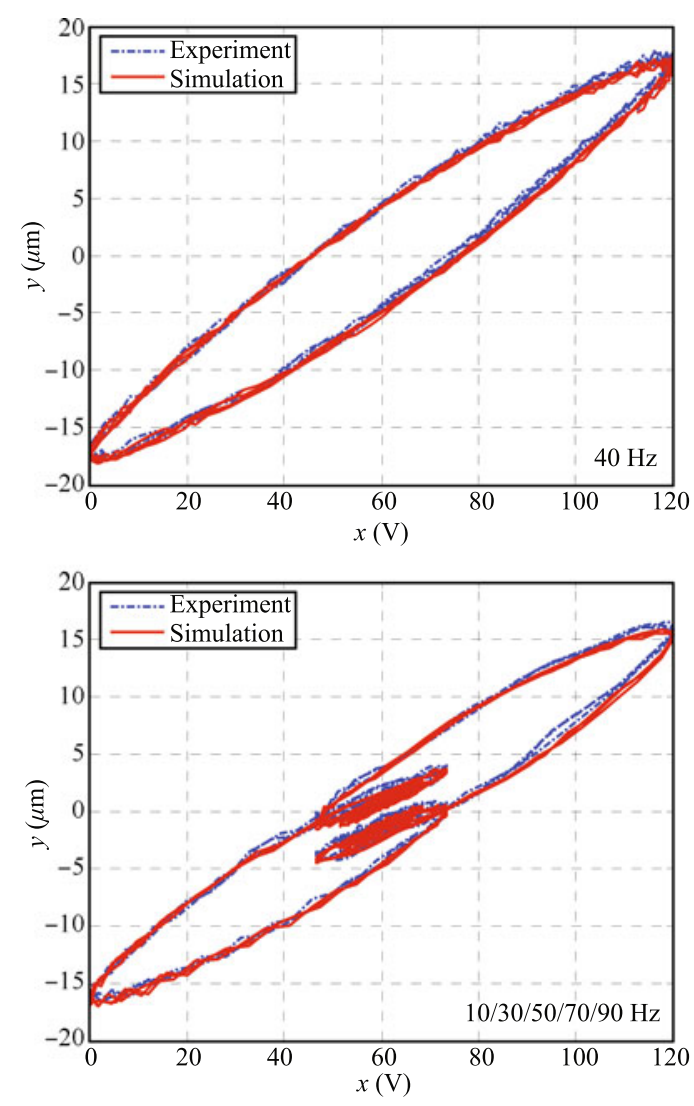

Fig. 4 Results of Hammerstein-like modeling

Table 1 Modeling results

\begin{tabular}{ccc}
\hline Frequency $(\mathrm{Hz})$ & ARMSE $(\mu \mathrm{m})$ & $\mathrm{RE}$ \\
\hline 1 & 0.3625 & 0.0215 \\
5 & 0.4958 & 0.0298 \\
10 & 0.5026 & 0.0304 \\
20 & 0.5132 & 0.0312 \\
30 & 0.5284 & 0.0335 \\
40 & 0.5492 & 0.0341 \\
50 & 0.5849 & 0.0352 \\
60 & 0.5926 & 0.0361 \\
70 & 0.6458 & 0.0388 \\
80 & 0.6534 & 0.0408 \\
90 & 0.7896 & 0.0519 \\
100 & 0.8169 & 0.0568 \\
$10 / 30 / 50 / 70 / 90$ & 0.9863 & 0.0672 \\
$30 / 60 / 90$ & 1.0638 & 0.0584 \\
$10 / \cdots / 90$ & 1.1259 & 0.0698 \\
\hline
\end{tabular}


It is noted that the $\mathrm{RE}$ in our testing frequency range of $1 \mathrm{~Hz}$ to $100 \mathrm{~Hz}$ can be ensured all less than $7 \%$ due to our modeling input signals frequency range from $1 \mathrm{~Hz}$ to $100 \mathrm{~Hz}$. To expand the applicable frequency range of the model, the modeling frequency range must also be expanded. Of course, it is expected that the $\mathrm{RE}$ will grow with the range of modeling frequency range.

\section{$3 \quad H_{\infty}$ robust controller design}

In order to improve the accuracy of the tracking control, a two-degree-of-freedom (2DOF) control scheme which can achieve good performance for both reference tracking and disturbance rejection is adopted, and an $H_{\infty}$ robust controller is designed based on the 2DOF control scheme. The rate-dependent hysteresis nonlinearity will first be compensated by its inverse nonlinear hysteresis model of the Hammerstein-like model, then the linear $H_{\infty}$ robust control theory is applied to the linear part ${ }^{[27,28]}$.

In Fig. $5, K$ is the $H_{\infty}$ robust controller, $W_{r}$ is the precompensation, $N$ is the nonlinear part of the Hammersteinlike model, $N^{-1}$ is the inverse model of nonlinear part, $L$ is the linear dynamic part of the Hammerstein-like model which is used as the nominal model, and $\tilde{L}$ represents the true linear part of PEA which can be defined as

$$
\tilde{L}=L+\Delta
$$

where $\Delta$ is the additive uncertainties of the nominal model which satisfies

$$
|\Delta(j \omega)| \leqslant\left|W_{\Delta}(j \omega)\right|, \quad \forall \omega \in \mathbf{R} .
$$

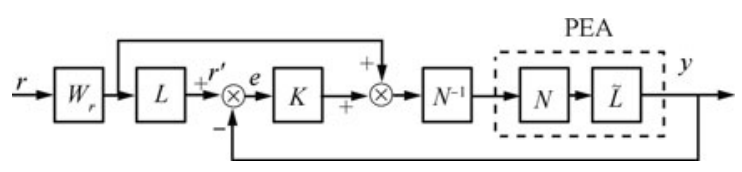

Fig. 5 2DOF control scheme

In this paper, $W_{\Delta}$ is obtained by the experiment according to Fig. 6.

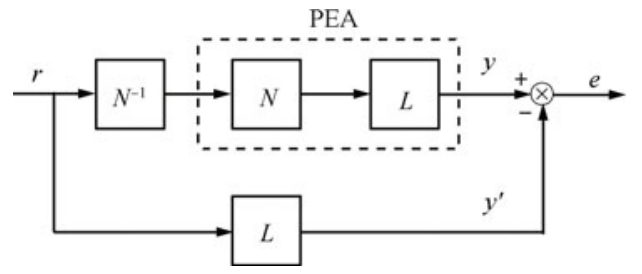

Fig. 6 The scheme of obtaining the uncertainty bound

Then we get the transfer function $W_{\Delta}$ as

$$
W_{\Delta}=\frac{0.07652(s+3.412)(s+46.25)}{(s+78.61)(s+7.846)} .
$$

Assume that $N^{-1}$ can compensate for the nonlinear part $N$, and take the uncompensated part as a linear uncertain system, then the control scheme can be replaced by the one with an additive uncertainty, as shown in Fig. 7.

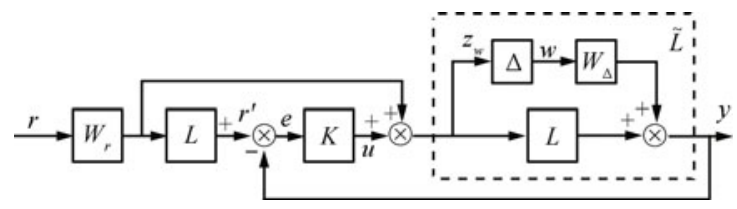

Fig. 7 2DOF control scheme with additive uncertainties

$W_{r}$ is chosen to be $L W_{r} \approx 1$ in order for $y$ to track the reference input $r$. In the Hammerstein-like modeling part, we get the linear model $L$ as

$$
L=\frac{0.2146 z-0.7352}{z^{2}-1.4215 z+1.0257} .
$$

Now let $W_{r}$ be

$$
\mathrm{W}_{r}=\frac{1}{z L}=\frac{z^{2}-1.4215 z+1.0257}{z(0.2146 z-0.7352)}
$$

In order to use continuous time $H_{\infty}$ robust control design method in the following, both $L$ and $W_{r}$ need to be transformed to continuous forms.

Then the problem can be transformed to a standard $H_{\infty}$ robust control problem in Fig. 8, where $P$ is the generalized plant and $K$ is the robust controller.

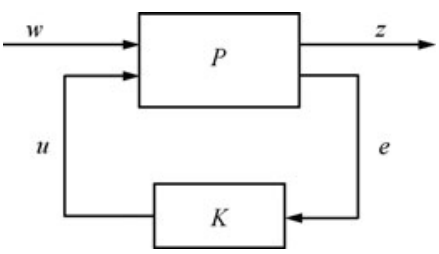

Fig. 8 The standard $H_{\infty}$ control system

The generalized input $w$ and output $z$ are defined as

$$
w=\left[\begin{array}{c}
w \\
r
\end{array}\right], \quad z=\left[\begin{array}{c}
z_{w} \\
e
\end{array}\right]
$$

and the generalized plant $P$ can be obtained from Fig. 7 as

$$
P=\left[\begin{array}{ccc}
0 & W_{r} & 1 \\
-W_{\Delta} & 0 & -L \\
-W_{\Delta} & 0 & -L
\end{array}\right]
$$

Let $T_{z w}$ denote the transfer function from $w$ to $z$. Then the problem is reduced to design an $H_{\infty}$ controller $K$ which satisfies

$$
\min _{K \text { stabilize } P}\left\|T_{z w}\right\|_{\infty}
$$

We apply the command hinfsyn in the Matlab Robust Toolbox to get a 7 th order controller $K_{7}$ and the command bstmr to get a 3rd order reduced-order controller $K_{3}$. Fig. 9 shows the Bode diagrams of the full-order and the reducedorder controllers.

$$
K_{3}=\frac{248.3778(s+1.329 \mathrm{e} 004)(s+2.227)}{s(s+3305)(s+174)} .
$$




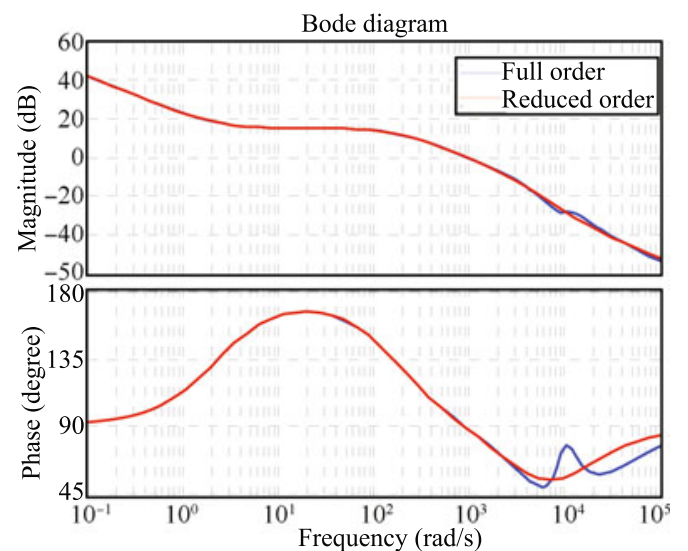

Fig.9 Bode diagram of the controllers (full-order and the reduced-order)

Both the amplitude and the phase frequency responses of the reduced-order robust controller do not change much from those of the full-order controller in the frequency range from $1 \mathrm{~Hz}$ to $100 \mathrm{~Hz}$ and beyond. Hence the reduced-order controller will be used in the tracking control experiment.

\section{Tracking control experiment}

The experimental setup was constructed to achieve trajectory tracking control as shown in Fig. 10. The PEA was manufactured by the Piezomechanik GmbH company, and the product type is PSt150/5/25 VS10. The stroke of the PEA is about $\pm 25 \mu \mathrm{m}$, and the applied voltage range of the PEA is $0-150 \mathrm{~V}$. The $\mathrm{D} / \mathrm{A}$ converter transforms the control signal from the industrial computer to the PEA by the specified power amplifier, and the displacement is measured by an eddy current sensor with a $0.1 \mu \mathrm{m}$ resolution. At the same time, the PEA displacement is transformed via the A/D converter to dSPACE control board (DS1103) and recorded in the industrial computer.

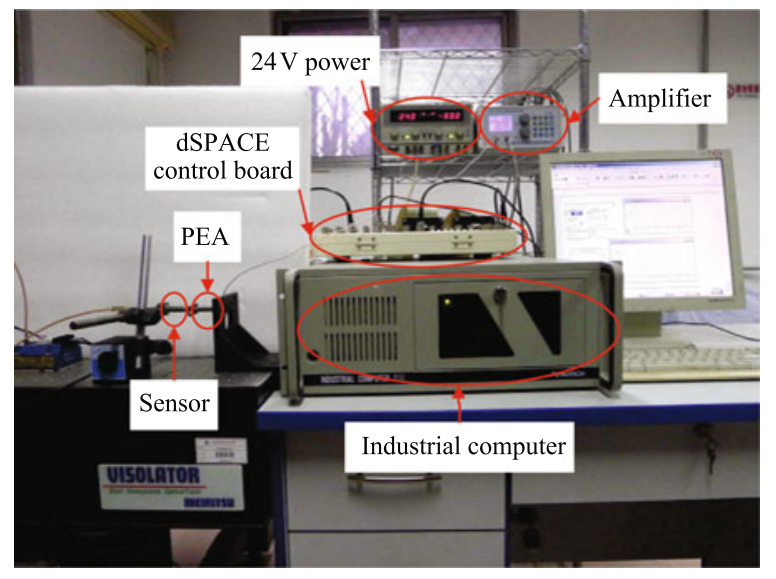

Fig. 10 Experiment equipment

We set the same amplitude $15 \mu \mathrm{m}$ for the different frequency input signals. The $H_{\infty}$ robust controller is designed based on the constructed Hammerstein-like model. It is connected in the system which can be used at different input signals either as a single-signal or a compound-signal.

Fig. 11 and Table 2 show the trajectory tracking control results of the $H_{\infty}$ robust controller at different single-signal and compound-signal inputs.
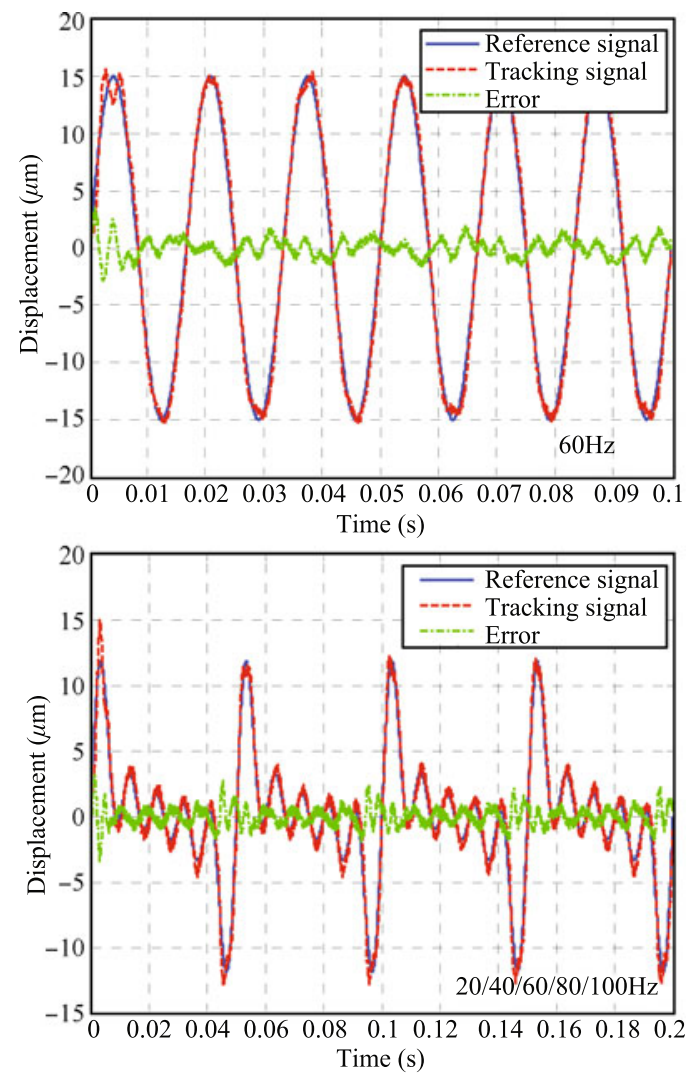

Fig. 11 Results of $H_{\infty}$ robust tracking control

Table 2 Tracking control results

\begin{tabular}{cccc}
\hline $\begin{array}{c}\text { Amplitude } \\
(\mu \mathrm{m})\end{array}$ & $\begin{array}{c}\text { Frequency } \\
(\mathrm{Hz})\end{array}$ & $\begin{array}{c}\text { ARMSE } \\
(\mu \mathrm{m})\end{array}$ & $\mathrm{RE}$ \\
\hline 15 & 1 & 0.2769 & 0.0247 \\
15 & 5 & 0.2764 & 0.0264 \\
15 & 10 & 0.2865 & 0.0271 \\
15 & 20 & 0.3285 & 0.0306 \\
15 & 30 & 0.3865 & 0.0321 \\
15 & 40 & 0.4315 & 0.0402 \\
15 & 50 & 0.5623 & 0.0518 \\
15 & 60 & 0.7269 & 0.0663 \\
15 & 70 & 0.8567 & 0.0741 \\
15 & 80 & 0.8972 & 0.0816 \\
15 & 90 & 0.9068 & 0.0884 \\
15 & 100 & 0.9684 & 0.0915 \\
15 & $10 / 20 / 40$ & 0.3659 & 0.0495 \\
15 & $10 / 30 / 50 / 70 / 90$ & 0.5676 & 0.0861 \\
15 & $20 / 40 / 60 / 80 / 100$ & 0.4012 & 0.0739 \\
\hline
\end{tabular}

Remark 1. The amplitude of reference signal should be constrained in the output driving range of the PEA between $u_{\min }$ and $u_{\max }$, otherwise the outranged reference signal may not be attained. To solve this problem, one method is to predict the output range of PEA during the modeling procedure.

Remark 2. The tracking control results in the above 
figure and table reveal that the $H_{\infty}$ robust controller is effective for both the single-input and the compound-input frequencies, and the maximum $\mathrm{RE}$ is less than $10 \%$ in the specified range of the PEA system.

\section{Conclusion}

This paper has proposed a rate-dependent hysteresis nonlinear model for precise tracking control of the PEA. We model the rate-dependent hysteresis by the Hammersteinlike model based on the SVM. Based on the Hammersteinlike model, we compensate the nonlinear part and design the $H_{\infty}$ robust controller for the rate-dependent linear model. Experimental results demonstrate that the Hammersteinlike model can capture the rate-dependent hysteresis effect, and the 2DOF control scheme with the $H_{\infty}$ robust controller for the rate-dependent nonlinear hysteresis is effective.

\section{References}

[1] J. Oh, D. S. Bernstein. Semilinear Duhem model for rateindependent and rate-dependent hysteresis. IEEE Transactions on Automatic Control, vol.50, no. 5, pp.631-645, 2005.

[2] Y. H. Yu, Z. C. Xiao, N. G. Naganathan, R. V. Dukkipati. Dynamic Preisach modelling of hysteresis for the piezoceramic actuator system. Mechanism and Machine Theory, vol. 37, no. 1, pp. 75-89, 2002.

[3] U. X. Tan, T. L. Win, C. Y. Shee, W. T. Ang. Ratedependent hysteresis model of piezoelectric using singularity free Prandtl-Ishlinskii model. In Proceedings of 2007 IEEE International Symposium on Computational Intelligence in Robotics and Automation, IEEE, Jacksonville, FL, USA, pp. 356-361, 2007.

[4] M. A. Janaideh, S. Rakheja, C. Y. Su. Experimental characterization and modeling of rate-dependent hysteresis of a piezoceramic actuator. Mechatronics, vol. 19, no. 5, pp. 656670, 2009.

[5] J. Oh, D. S. Bernstein. Identification of rate-dependent hysteresis using the semilinear Duhem model. In Proceedings of 2004 American Control Conference, IEEE, Boston, MA, USA, pp. 4776-4781, 2004.

[6] J. Oh, D. S. Bernstein. Piecewise linear identification for the rate-independent and rate-dependent Duhem hysteresis models. IEEE Transactions on Automatic Control, vol. 52, no. 3, pp. 576-582, 2005.

[7] R. L. Dong, Y. H. Tan, H. Chen, Y. Q. Xie. A neural networks based model for rate-dependent hysteresis for piezoceramic actuators. Sensors and Actuators A: Physical, vol. 143, no. 2, pp. 370-376, 2008.

[8] J. P. Lien, A. York, T. G. Fang, G. D. Buckner. Modeling piezoelectric actuators with hysteretic recurrent neural networks. Sensors and Actuators A: Physical, vol. 163, no. 2, pp. 516-525, 2010.
[9] J. Q. Mao, H. S. Ding. Intelligent modeling and control for nonlinear systems with rate-dependent hysteresis. Science in China (Series F: Information Sciences), vol. 52, no. 4, pp. 656-673, 2009.

[10] R. C. Smith. Inverse compensation for hysteresis in magnetostrictive transducers. Mathematical and Computer Modelling, vol. 33, no. 1-3, pp. 285-298, 2001.

[11] X. L. Zhang, Y. H. Tan, M. Y. Su, Y. Q. Xie. Neural networks based identification and compensation of ratedependent hysteresis in piezoelectric actuators. Physica B: Condensed Matter, vol. 405, no. 12, pp. 2687-2693, 2010.

[12] X. K. Chen, T. Hisayama, C. Y. Su. Pseudo-inverse-based adaptive control for uncertain discrete time systems preceded by hysteresis. Automatica, vol. 45, no. 2, pp. 469-476, 2009.

[13] Y. Feng, C. A. Rabbath, H. Hong, M. A. Janaideh, C. Y. Su. Robust control for shape memory alloy micro-actuators based flap positioning system. In Proceedings of the American Control Conference, IEEE, Baltimore, MD, USA, pp. 4181-4186, 2010.

[14] W. S. Oates, R. C. Smith. Nonlinear optimal control techniques for vibration attenuation using magnetostrictive actuators. Journal of Intelligent Material Systems and Structures, vol. 19, no. 2, pp. 193-209, 2008.

[15] E. Eskinat, S. H. Johnson, W. L. Luyben. Use of Hammerstein models in identification of nonlinear systems. AIChE Journal, vol. 37, no. 2, pp. 255-268, 1991.

[16] Y. Rochdi, F. Giri, J. B. Gning, F. Z. Chaoui. Identification of block-oriented systems in the presence of nonparametric input nonlinearities of switch and backlash types. Automatica, vol. 46, no. 5, pp. 864-877, 2010.

[17] Z. H. Lang. Controller design oriented model identification method for Hammerstein system. Automatica, vol. 29, no. 3 , pp. $767-771,1993$.

[18] M. Verhaegen, D. Westwick. Identifying MIMO Hammerstein systems in the context of subspace model identification methods. International Journal Control, vol.63, no. 2, pp. 331-349, 1996.

[19] G. Golub, V. Pereyra. Separable nonlinear least squares: The variable projection method and its applications. Inverse Problems, vol. 19, no. 2, pp. R1-R26, 2003.

[20] E. W. Bai, M. Y. Fu. A blind approach to Hammerstein model identification. IEEE Transactions on Signal Processing, vol. 50, no. 7, pp. 1610-1619, 2002.

[21] F. Ding, T. Chen. Gradient based iterative algorithms for solving a class of matrix equations. IEEE Transactions on Automatic Control, vol. 50, no. 8, pp. 1216-1221, 2005.

[22] Y. Rochdi, F. Giri, J. B. Gning, F. Z. Chaoui. Identification of block-oriented systems in the presence of nonparametric input nonlinearities of switch and backlash types. Automatica, vol. 46, no. 5, pp. 864-877, 2010. 
[23] V. N. Vapnik. The Nature of Statistical Learning Theory, New York, USA: Springer-Verlag, 1995.

[24] Y. H. Shi, R. C. Eberhart. Empirical study of particle swarm optimization. In Proceedings of 1999 Congress on Evolutionary Computation, IEEE, Washington, DC, USA, pp. 1945-1950, 1999.

[25] J. Q. Mao, H. S. Ding, Y. H. Ma. Fuzzy dynamic model of a giant magnetostrictive actuator. Materials Science Forum, vol. 546-549, pp. 2183-2188, 2007.

[26] L. Ljung. System Identification: Theory for the User, 2nd ed., Upper Saddle River, N J: PTR Prentice Hall, 1999.

[27] K. M. Zhou, J. C. Doyle, K. Glover. Robust and Optimal Control, Upper Saddle River, New Jersey: Prentice-Hall, 1996.

[28] K. M. Zhou, J. C. Doyle. Essentials of Robust Control, Upper Saddle River, New Jersey: Prentice Hall, 1997.

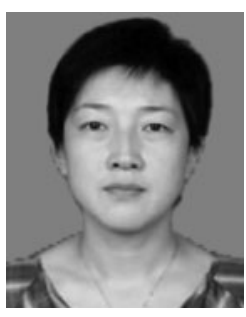

system.

E-mail: llpp0203@163.com (Corresponding author)

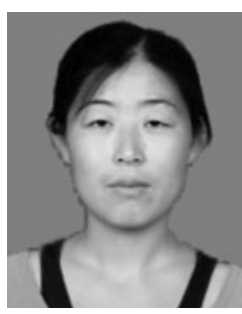

Zhen-Yan Wang received her M. Eng. degree in system engineering from Taiyuan University of Science and Technology, China in 2005. Since 2002, she has been with the School of Electronics and Information Engineering, Taiyuan University of Science and Technology. She is currently a $\mathrm{Ph} . \mathrm{D}$. candidate at the School of $\mathrm{Au}-$ tomation Science and Electrical Engineering, Beijing University of Aeronautics and

Astronautics, China.

Her research interests include modeling and control of dynamic hysteresis nonlinear system.

E-mail: w9851@126.com

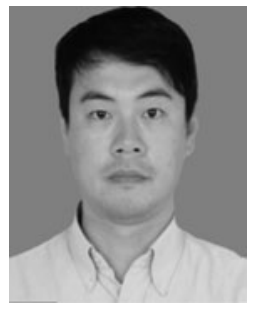

Zhen Zhang received his $\mathrm{Ph} . \mathrm{D}$. degree in solid mechanics from Xi'an Jiaotong University, China in 2004. His thesis concerned the characterization and modeling of shape memory alloy structures. From 2004 to 2006, he worked as postdoctoral in Beijing University of Aeronautics and Astronautics, China. Since 2006, he has been with the School of Automation Sciences and Electrical Engineering, Beijing University of Aeronautics and Astronautics.

His research interests include dynamics and control of smart structures.

E-mail: zhangzhen@buaa.edu.cn

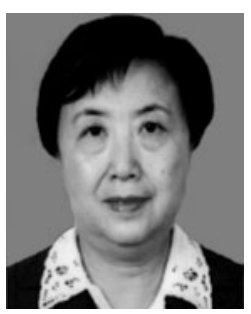

Jian-Qin Mao received her Ph. D. degree in aeronautical navigation and control engineering from Beijing University of Aeronautics and Astronautics, China in 1985. Her major field of study concerned the fuzzy modeling and control with applications, and smart structure dynamics and control. She is professor at the School of Automation Science and Electrical Engineering, Beijing University of Aeronautics

and Astronautics.

Her research interests include the area of dynamics and control of smart structures and smart actuator.

E-mail: jqmao@buaa.edu.cn

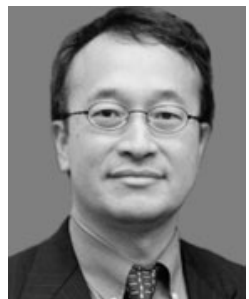

Ke-Min Zhou received his B. Sc. degree from Beijing University of Aeronautics and Astronautics, China in 1982, M. Sc. E.E. and $\mathrm{Ph}$. D. degrees from University of Minnesota in 1986 and 1988, respectively. Since 1990, he has been with Louisiana State University where he is currently Roy Paul Daniels Distinguished Professor and Mark and Carolyn C. Guidry Professor in electrical and computer engineering. He has a joint appointment with Southwest Jiaotong University. He is the leading author of two books in the field: Robust and Optimal Control (Prentice Hall, 1995) and Essentials of Robust Control (Prentice Hall, 1997). He is also an author of the book Introduction to Feedback Control by Pearson Prentice Hall in 2009. $\mathrm{He}$ is/was an associate editor of Automatica, IEEE Transactions on Automatic Control, SIAM Journal on Control and Optimization, Systems and Control Letters, Journal of System Sciences and Complexity, and Journal of Control Theory and Applications. He is a fellow of IEEE and a fellow of AAAS.

E-mail: kmzhou@gmail.com 